\title{
SOME REMARKS ON DERIVATIONS IN SEMIPRIME RINGS AND STANDARD OPERATOR ALGEBRAS
}

\author{
JOSO VUKMAN \\ University of Maribor, Slovenia
}

\begin{abstract}
In this paper identities related to derivations on semiprime rings and standard operator algebras are investigated. We prove the following result which generalizes a classical result of Chernoff. Let $X$ be a real or complex Banach space, let $L(X)$ be the algebra of all bounded linear operators of $X$ into itself and let $A(X) \subseteq L(X)$ be a standard operator algebra. Suppose there exists a linear mapping $D: A(X) \rightarrow L(X)$ satisfying the relation $2 D\left(A^{3}\right)=D\left(A^{2}\right) A+A^{2} D(A)+D(A) A^{2}+A D\left(A^{2}\right)$ for all $A \in A(X)$. In this case $D$ is of the form $D(A)=A B-B A$ for all $A \in A(X)$ and some fixed $B \in L(X)$, which means that $D$ is a linear derivation.
\end{abstract}

This research has been motivated by the work of Brešar ([3]) and Chernoff ([4]) and it is a continuation of our recent work ([11-13]). Throughout, $R$ will represent an associative ring with center $Z(R)$. As usual we write $[x, y]$ for $x y-y x$. Given an integer $n \geq 2$, a ring $R$ is said to be $n$-torsion free, if for $x \in R, n x=0$ implies $x=0$. Recall that a ring $R$ is prime if for $a, b \in R, a R b=(0)$ implies $a=0$ or $b=0$, and semiprime in case $a R a=(0)$ implies $a=0$. Let $A$ be an algebra over the real or complex field and let $B$ be a subalgebra of $A$. A linear mapping $D: B \rightarrow A$ is called a linear derivation in case $D(x y)=D(x) y+x D(y)$ holds for all pairs $x, y \in R$. In case we have a ring $R$ an additive mapping $D: R \rightarrow R$ is called a derivation if $D(x y)=D(x) y+x D(y)$ holds for all pairs $x, y \in R$ and is called a Jordan derivation in case $D\left(x^{2}\right)=D(x) x+x D(x)$ is fulfilled for all $x \in R$. A derivation $D$ is inner in case there exists $a \in R$ such that $D(x)=[x, a]$ holds for all $x \in R$. Every derivation is a Jordan derivation. The converse is in

2010 Mathematics Subject Classification. 16W10, 46K15, $39 \mathrm{~B} 05$.

Key words and phrases. Prime ring, semiprime ring, Banach space, standard operator algebra, derivation, Jordan derivation, Jordan triple derivation.

This research has been supported by the Research Council of Slovenia. 
general not true. A classical result of Herstein ([6]) asserts that any Jordan derivation on a 2 -torsion free prime ring is a derivation. A brief proof of Herstein's result can be found in [1]. Cusack ([5]) generalized Herstein's result to 2-torsion free semiprime rings (see also [2] for an alternative proof). An additive mapping $D: R \rightarrow R$, where $R$ is an arbitrary ring, is called a Jordan triple derivation in case $D(x y x)=D(x) y x+x D(y) x+x y D(x)$ holds for all pairs $x, y \in R$. One can easily prove that any Jordan derivation $D$ on an arbitrary 2 - torsion free ring $R$ is a Jordan triple derivation (see, for example, $[1])$. Let $X$ be a real or complex Banach space and let $L(X)$ and $F(X)$ denote the algebra of all bounded linear operators on $X$ and the ideal of all finite rank operators in $L(X)$, respectively. An algebra $A(X) \subseteq L(X)$ is said to be standard in case $F(X) \subseteq A(X)$. Let us point out that any standard operator algebra is prime, which is a consequence of Hahn-Banach theorem.

Let us start with the following result proved by Brešar ([3]).

THEOREM 1. Let $R$ be a 2 -torsion free semiprime ring and let $D: R \rightarrow R$ be a Jordan triple derivation. In this case $D$ is a derivation.

Since, as we mentioned above, any Jordan derivation $D$ on arbitrary $2-$ torsion free ring is a Jordan triple derivation, one can conclude that Theorem 1 generalizes Cusack's generalization of Herstein's theorem. We proceed with the following result which is motivated by Theorem 1 .

TheOREM 2. Let $R$ be a 2 -torsion free semiprime ring and let $D: R \rightarrow R$ be an additive mapping. Suppose that either

$$
D(x y x)=D(x y) x+x y D(x)
$$

$$
D(x y x)=D(x) y x+x D(y x)
$$

holds for all pairs $x, y \in R$. In both cases $D$ is a derivation.

The approach we use in the proof of Theorem 2 differs from those used by Brešar in his proof of Theorem 1. For the proof of Theorem 2 we need the lemma bellow (see [10, Lemma 3]).

Lemma 3. Let $R$ be a semiprime ring and let $f: R \rightarrow R$ be an additive mapping. Suppose that either

$$
f(x) x=0
$$

or

$$
x f(x)=0
$$

holds for all $x \in R$. In both cases $f=0$.

Proof of Theorem 2. Let us assume that the relation (1) is fulfilled. The linearization of the relation (1) gives

$$
D(x y z+z y x)=D(x y) z+D(z y) x+x y D(z)+z y D(x)
$$


for all $x, y, z \in R$. In particular, for $z=x^{2}$ the above relation gives

(3) $D\left(x y x^{2}+x^{2} y x\right)=D(x y) x^{2}+D\left(x^{2} y\right) x+x y D\left(x^{2}\right)+x^{2} y D(x), x, y \in R$.

The substitution $x y+y x$ for $y$ in the relation (1) gives

$$
D\left(x y x^{2}+x^{2} y x\right)=D\left(x^{2} y\right) x+D(x y x) x+x^{2} y D(x)+x y x D(x), x, y \in R .
$$

We have therefore using (1)

(4) $D\left(x y x^{2}+x^{2} y x\right)=D\left(x^{2} y\right) x+D(x y) x^{2}+x y D(x) x+x^{2} y D(x)+x y x D(x)$ for all pairs $x, y \in R$. By comparing (3) and (4) we obtain

$$
x y A(x)=0
$$

for all pairs $x, y \in R$, where $A(x)$ stands for $D\left(x^{2}\right)-D(x) x-x D(x)$. Right multiplication of (5) by $x$ and left multiplication by $A(x)$ gives $A(x) x y A(x) x=0$ for all pairs $x, y \in R$, whence it follows

$$
A(x) x=0
$$

for all $x \in R$ by semiprimeness of $R$. The substitution $A(x) y x$ for $y$ in the relation (5) gives $x A(x) y x A(x)=0$ for all pairs $x, y \in R$, which gives

$$
x A(x)=0
$$

for all $x \in R$. The linearization of the relation (6) gives

$$
B(x, y) x+A(x) y+B(x, y) y+A(y) x=0
$$

for all pairs $x, y \in R$, where $B(x, y)$ denotes $D(x y+y x)-D(x) y-x D(y)-$ $D(y) x-y D(x)$. Putting in the above relation $-x$ for $x$ and comparing the relation so obtained with the above relation one obtains

$$
B(x, y) x+A(x) y=0
$$

for all pairs $x, y \in R$. Right multiplication of the above relation by $A(x)$ gives, because of the relation (7), $A(x) y A(x)=0$ for all pairs $x, y \in R$, whence it follows $A(x)=0$ for all $x \in R$. In other words, $D$ is a Jordan derivation. By Cusack's generalization of Herstein's theorem one can conclude that $D$ is a derivation. In [3] Brešar has proved Theorem 1 without using Cusack's generalization of Herstein's theorem. It is our aim to show that Theorem 2 can be proved without using Cusack's generalization of Herstein's theorem as well. From the fact that $D$ is a Jordan derivation it follows that $D$ is a Jordan triple derivation. Now, comparing the relation $D(x y x)=$ $D(x) y x+x D(y) x+x y D(x), x, y \in R$, with the relation (1) one obtains

$$
(D(x y)-D(x) y-x D(y)) x=0, x, y \in R .
$$

For any fixed $y \in R$ we have an additive mapping $x \mapsto D(x y)-D(x) y-x D(y)$ on $R$. Thus from the above relation and Lemma 3 it follows $D(x y)-D(x) y-$ $x D(y)=0$ for all pairs $x, y \in R$. In other words, $D$ is a derivation. The proof 
that $D$ is a derivation in case $D(x y x)=D(x) y x+x D(y x)$ holds for all pairs $x, y \in R$ goes through in a similar way and will therefore be omitted.

Disadvantage of Theorem 2 is that in identities (1) and (2) there is no symmetry. Theorem 2 together with the desire for symmetry leads to the following conjecture.

Conjecture 4. Let $R$ be a 2 -torsion free semiprime ring and let $D$ : $R \rightarrow R$ be an additive mapping. Suppose that

$$
2 D(x y x)=D(x y) x+x y D(x)+D(x) y x+x D(y x)
$$

holds for all pairs $x, y \in R$. In this case $D$ is a derivation.

Our next result is in the spirit of the conjecture above.

Theorem 5. Let $X$ be a real or complex Banach space and let $A(X)$ be a standard operator algebra on $X$. Suppose there exists a linear mapping $D: A(X) \rightarrow L(X)$ satisfying the relation

$$
2 D\left(A^{3}\right)=D\left(A^{2}\right) A+A^{2} D(A)+D(A) A^{2}+A D\left(A^{2}\right)
$$

for all $A \in A(X)$. In this case $D$ is of the form $D(A)=[A, B]$ for all $A \in$ $A(X)$ and some fixed $B \in L(X)$, which means that $D$ is a linear derivation.

Theorem 5 generalizes the result below first proved by Chernoff ([4]) (see also $[8,9])$.

TheOREM 6. Let $X$ be a real or complex Banach space, let $A(X)$ be a standard operator algebra on $X$ and let $D: A(X) \rightarrow L(X)$ be a linear derivation. In this case $D$ is of the form $D(A)=[A, B]$ for all $A \in A(X)$ and some fixed $B \in L(X)$.

In the proof of Theorem 5 we use Herstein's theorem, Theorem 6 and methods which are similar to those used in [11-13].

Proof of TheOrem 5. We have therefore the relation

$$
2 D\left(A^{3}\right)=D\left(A^{2}\right) A+A^{2} D(A)+D(A) A^{2}+A D\left(A^{2}\right)
$$

for all $A \in A(X)$. Let us first consider the restriction of $D$ on $F(X)$. Let $A$ be from $F(X)$ and let $P \in F(X)$ be a projection with $A P=P A=A$. Putting $A+P$ for $A$ in the relation (8) we obtain after some calculations

$$
\begin{aligned}
6 D\left(A^{2}\right)+6 D(A)= & 4 D(A) A+4 A D(A)+D\left(A^{2}\right) P+P D\left(A^{2}\right) \\
& +D(P) A^{2}+A^{2} D(P)+3 D(A) P+3 P D(A) \\
& +3 A D(P)+3 D(P) A .
\end{aligned}
$$

Putting in the above relation $-A$ for $A$ and comparing the relation so obtained with the above relation we obtain

(9) $6 D\left(A^{2}\right)=4 D(A) A+4 A D(A)+D\left(A^{2}\right) P+P D\left(A^{2}\right)+D(P) A^{2}+A^{2} D(P)$ 
and

$$
2 D(A)=D(A) P+P D(A)+A D(P)+D(P) A .
$$

Putting $A^{2}$ for $A$ in the relation (10) we obtain

$$
2 D\left(A^{2}\right)=D\left(A^{2}\right) P+P D\left(A^{2}\right)+A^{2} D(P)+D(P) A^{2}
$$

which reduces the relation (9) to

$$
D\left(A^{2}\right)=D(A) A+A D(A) .
$$

The relation (11) is fulfilled for any $A \in F(X)$. From the relation (10) one can conclude that $D$ maps $F(X)$ into itself. We have therefore a linear mapping which maps $F(X)$ into itself satisfying the relation (11) for all $A \in F(X)$, which means that $D$ is a Jordan derivation on $F(X)$. Since $F(X)$ is prime it follows that $D$ is a derivation by Herstein's theorem. Applying Theorem 6 one can conclude that $D$ is of the form

$$
D(A)=[A, B]
$$

for all $A \in F(X)$ and some fixed $B \in L(X)$. It remains to prove that the relation (12) holds for all $A \in A(X)$ as well. For this purpose we introduce $D_{1}: A(X) \rightarrow L(X)$ by $D_{1}(A)=[A, B]$ and consider $D_{0}=D-D_{1}$. The mapping $D_{0}$ is, obviously, linear and satisfies the relation (8). Besides, $D_{0}$ vanishes on $F(X)$. It is our aim to prove that $D_{0}$ vanishes on $A(X)$ as well. Let $A \in A(X)$, let $P$ be an one-dimensional projection and let us introduce $S \in A(X)$ by $S=A+P A P-(A P+P A)$. We have $S P=P S=0$. It is easy to see that $D_{0}(S)=D_{0}(A)$ and $D_{0}\left(S^{2}\right)=D_{0}\left(A^{2}\right)$. Now we have

$$
\begin{aligned}
& D_{0}\left(S^{2}\right) S+S^{2} D_{0}(S)+D_{0}(S) S^{2}+S D_{0}\left(S^{2}\right) \\
& =2 D_{0}\left(S^{3}\right)=2 D_{0}\left(S^{3}+P\right)=2 D_{0}\left((S+P)^{3}\right) \\
& =D_{0}\left(S^{2}\right)(S+P)+\left(S^{2}+P\right) D_{0}(S)+D_{0}(S)\left(S^{2}+P\right)+(S+P) D_{0}\left(S^{2}\right) .
\end{aligned}
$$

We have therefore $D_{0}\left(S^{2}\right) P+P D_{0}(S)+D_{0}(S) P+P D_{0}\left(S^{2}\right)=0$ and since $D_{0}(S)=D_{0}(A)$ and $D_{0}\left(S^{2}\right)=D_{0}\left(A^{2}\right)$ we arrive at

$$
D_{0}\left(A^{2}\right) P+P D_{0}(A)+D_{0}(A) P+P D_{0}\left(A^{2}\right)=0 .
$$

Putting in the above relation $-A$ for $A$ and comparing the relation so obtained with the relation (13) we obtain

$$
P D_{0}(A)+D_{0}(A) P=0 .
$$

Multiplying the above relation from both sides by $P$ we obtain

$$
P D_{0}(A) P=0 \text {. }
$$

Right multiplication of the relation (14) by $P$ gives, because of (15),

$$
D_{0}(A) P=0 \text {. }
$$


Since $P$ is an arbitrary one-dimensional projection we have $D_{0}(A)=0$ for all $A \in A(X)$, which was our intension to prove. The proof of the theorem is complete.

In the proof of Theorem 5 we used some ideas similar to those used by Molnár in [7].

ACKNOWLEDGEMENTS.

The author wishes to express his thanks to referees for helpful comments.

\section{REFERENCES}

[1] M. Brešar and J. Vukman, Jordan derivations on prime rings, Bull. Austral. Math. Soc. 37 (1988), 321-322.

[2] M. Brešar, Jordan derivations on semiprime rings, Proc. Amer. Math. Soc. 104 (1988), 1003-1006.

[3] M. Brešar, Jordan mappings of semiprime rings, J. Algebra 127 (1989), 218-228.

[4] P. R. Chernoff, Representations, automorphisms, and derivations of some operator algebras, J. Functional Analysis 12 (1973), 275-289.

[5] J. Cusack, Jordan derivations on rings, Proc. Amer. Math. Soc. 53 (1975), 321-324.

[6] I. N. Herstein, Jordan derivations of prime rings, Proc. Amer. Math. Soc. 8 (1957), 1104-1110.

[7] L. Molnár, On centralizers of an $H^{*}$-algebra, Publ. Math. Debrecen 46 (1995), 89-95.

[8] P. Šemrl, Ring derivations on standard operator algebras, J. Funct. Anal. 112 (1993), $318-324$.

[9] J. Vukman, On automorphisms and derivations of operator algebras, Glas. Mat. Ser. III 19 (1984), 135-138.

[10] J. Vukman, Identities with derivations and automorphisms on semiprime rings, Int. J. Math. Math. Sci. (2005), 1031-1038.

[11] J. Vukman, On derivations of algebras with involution, Acta Math. Hungar. 112 (2006), 181-186.

[12] J. Vukman, On derivations of standard operator algebras and semisimple $H^{*}$-algebras, Studia Sci. Math. Hungar. 44 (2007), 57-63.

[13] J. Vukman, Identities related to derivations and centralizers on standard operator algebras, Taiwanese J. Math. 11 (2007), 255-265.

J. Vukman

Department of Mathematics and Computer Science

University of Maribor

FNM, Koroška 160, 2000 Maribor

Slovenia

E-mail: joso.vukman@uni-mb.si

Received: 11.8.2009.

Revised: 21.1.2010. \& 9.2.2010. 